\title{
Novos paradigmas para avaliação da qualidade da informação em saúde recuperada na $W e b$
}

\section{Ilza Leite Lopes}

Doutoranda e pesquisadora associada na UnB/CID. Professora na AEUDF.

E-mail: ilzall@terra.com.br

ilzall@unb.br

\section{Resumo}

A revolução nos paradigmas de produção do conhecimento e as implicações na qualidade de informação disponibilizadas na World Wide Web (Web) são analisadas. Apresentam-se as questões relacionadas com os critérios de avaliação da qualidade da informação em saúde recuperadas na $\mathbf{W e b}$, com ênfase naqueles definidos pela Agency for Health Care Policy and Research (AHCPR), do Health Information Technology Institute (Hiti). Destacam-se os estudos e as iniciativas internacionais desenvolvidas com o objetivo de avaliar, controlar a assegurar a qualidade da informação em saúde na Web e as propostas de instituições brasileiras quanto a esta questão.

\section{Palavras-chave}

Qualidade de informação em Saúde; Critérios de avaliação; Recuperação da informação na Web; Iniciativas internacionais; Propostas brasileiras.

\section{New paradigms for evaluation of the information quality health retrieved on the Web}

\begin{abstract}
The revolution in the paradigms of the knowledge production and the implications in the information quality available on the World Wide Web (Web) are analyzed. Presents the questions related with the evaluation criteria of the information quality health retrieved in the Web, focusing on the criteria defined by the Agency for Health Care Policy and Research (AHCPR), of the Health Information Technology Institute (Hiti). To point out the studies and the international initiatives developed with the objective to evaluate, to control and to assure the quality of Health information in the Web and the proposals of Brazilian institutions concerning these questions.
\end{abstract}

Keywords

Health information quality; Evaluation criteria; Information retrieval in the Web; International initiatives; Brazilian proposals.

\section{INTRODUÇÃO}

O processo de recuperação da informação encontra-se diretamente relacionado com o crescimento exponencial de publicações eletrônicas disponibilizadas na World Wide Web (Web), uma aplicação da Internet que ganhou proeminente lugar entre as outras aplicações como Telnet, File Transfer Protocol (FTP), Correio Eletrônico (e-mail), Gopher, por conta de sua interface amigável para o usuário e de sua característica de hipermídia, tornandose amplo repositório de dados em contínuo crescimento, mudando os paradigmas da produção científica.

As profundas mudanças que a Internet vem provocando no modelo de produção e disseminação da informação têm sido consideradas na literatura especializada, segundo atesta Jones (2000), como uma revolução dos paradigmas no modelo até então vigente, pois o distanciamento observado entre o modelo anterior ao advento da Internet e da Web tomou proporções gigantescas em relação ao modelo observado atualmente.

Kuhn (1975) demonstrou que um paradigma é o que os membros de uma comunidade científica compartilham. $\mathrm{O}$ aspecto mais importante de sua teoria focaliza a qualidade da informação eletrônica recuperada na Web, e, do contexto deste trabalho, destaca-se a ênfase dada ao caráter revolucionário do próprio progresso científico, que ocorre mediante saltos, e não em linha contínua. Estes saltos, preconizados por Kuhn, ocorrem nos períodos de desenvolvimento científico em que são questionados os princípios, as teorias, os conceitos básicos e as metodologias que até então orientavam toda a investigação e toda a prática científica de disseminação de novos conhecimentos.

Neste sentido, constata-se que estamos diante de um novo paradigma, no que se refere à manipulação da informação em todos os seus estágios, desde o emissor até o receptor. As publicações eletrônicas disponibilizadas na Web constituem um dos temas de maior repercussão dentro da comunidade científica, permitindo o acesso, produção e disseminação de informação em larga escala, por um único indivíduo ou por organizações, revolucionando toda a estrutura desta produção, disseminação e acesso que estava em vigor antes do advento da Internet. 


\section{CRITÉRIOS DE AVALIAÇÃO DA QUALIDADE DA INFORMAC̣ÃO NA WEB}

Os novos paradigmas da chamada sociedade da informação, com seus imperativos informacionais, agregam diariamente novas páginas na Web, de caráter individual, de associações, de grupos de interesse, de instituições privadas, governamentais e outras, de forma acelerada e irrestrita. Este senso de urgência provocou o desenvolvimento não-ordenado e não-planejado de páginas Web, com informação de todo o tipo, não necessariamente científica.

Considerando que a ciência é dependente do presente sistema de comunicação para a produção e o acesso à informação, convém relembrar que o processo de produção do conhecimento anterior à Web incorporava rigoroso mecanismo de controle de qualidade. A revisão dos trabalhos científicos pelos pares antecedia a publicação e a divulgação destes trabalhos, e, na prática, este procedimento era considerado, na comunidade científica, como um qualificador para a disseminação e legitimação do conhecimento. A revisão efetuada, a pedido de um editor de publicação científica, aos especialistas de determinada área assegurava o anonimato do revisor e, ao mesmo tempo, garantia a acurácia e a qualidade do texto apresentado para publicação.

O advento da tecnologia da informação aplicada ao processo de divulgação de trabalhos científicos proporcionou maiores facilidades para a revisão e a validação de textos por meios eletrônicos de forma mais dinâmica, mantendo, porém, os mesmos princípios reguladores de qualidade dos trabalhos científicos a serem divulgados. Cabe ressaltar, no entanto, que o sentido da palavra publicação, em termos eletrônicos, tem atualmente um novo significado com estas tecnologias, possibilitando, a qualquer pessoa com acesso à Internet, a exposição dos seus trabalhos com escasso ou nulo controle profissional sobre o que se está apresentando, afetando, conseqüentemente, a qualidade do conteúdo desta informação.

Entretanto, a qualidade da informação é um dos mais importantes aspectos a serem considerados, devido ao volume exponencialmente crescente de informações veiculadas na Internet, sendo que, para os consumidores, os conteúdos das páginas institucionais ou de quaisquer documentos que são disponibilizados necessitam de filtros para minimizar o excesso de informação tornada disponível. Como determinar a qualidade dessa informação descentralizada e flutuante? Buscando a solução deste problema, desde 1995, especialistas em

\section{QUADRO 1 \\ Critérios categorizados como indicadores de qualidade do HSWG}

\begin{tabular}{l|l}
\hline Categoria & Indicadores de qualidade \\
\hline 1. Credibilidade & $\begin{array}{l}\text { a) Fonte, b) Contexto, c) Atualização, } \\
\text { d) Pertinência/ Utilidade, e) Processo de } \\
\text { Revisão Editorial }\end{array}$ \\
2. Conteúdo & $\begin{array}{l}\text { a) Acurácia, b) Hierarquia de evidência, } \\
\text { c) Precisão das fontes, d) Avisos } \\
\text { institucionais, e) Completeza }\end{array}$ \\
3. Apresentação do & a) objetivo, b) Perfil do site \\
site & $\begin{array}{l}\text { a) Seleção, b) Arquitetura, c) Conteúdo, } \\
\text { d) Links de retorno }\end{array}$ \\
5. Design & $\begin{array}{l}\text { a) Acessibilidade, b) Navegabilidade, } \\
\text { c) Mecanismo de busca interno }\end{array}$ \\
6. Interatividade & $\begin{array}{l}\text { a) Mecanismo de retorno da informação, } \\
\text { b) Fórum de discussão, c) Explicitação de } \\
\text { algoritmos }\end{array}$ \\
7. Anúncios & a) Alertas \\
\hline
\end{tabular}

Fonte: http://hitiweb.mitretek.org/docs/policy.html

informação têm apresentado, de forma independente, critérios ou filtros de qualidade para avaliação das páginas da Web. A idéia central desses filtros ou critérios é a mesma, isto é, as listas contêm categorias e subcategorias de problemas críticos relacionados com a qualidade da informação da Web e, normalmente, identificam esses critérios com as categorias apresentadas para a área de saúde pela organização americana Agency for Health Care Policy and Research (AHCPR), no Health Information Technology Institute (Hiti), por intermédio do Grupo de Trabalho Health Summit Working Group (HSWG), que definem as seguintes categorias: credibilidade, conteúdo, apresentação formal do site, links, design, interatividade e anúncios, conforme apresentado no quadro 1.

Para a determinação da credibilidade da informação em saúde na Web, foram estabelecidos, pela agência, os seguintes elementos: a fonte de informação médica, como o primeiro indicador para estabelecer a credibilidade, implicando a visualização da logomarca e do nome da instituição ou do responsável pela informação, assim como o nome e a titulação do autor. Por este critério, devem ser registrados os componentes de atualidade da 
informação, da relevância e, ainda, o processo de revisão editorial nos textos disseminados na Web.

Em relação ao critério de conteúdo, a agência definiu alguns elementos básicos para serem avaliados: precisão e acurácia da informação, hierarquia de evidência, quadros de avisos descrevendo as limitações, objetivos, cobertura, autoridade e atualidade da informação, todos descritos em linguagem acessível não apenas aos profissionais de saúde, mas especialmente para os leigos. Define ainda como necessárias a precisão das fontes e a completeza da informação, destacando que o tratamento de determinado tema deve ser compreensível e balanceado. Assim, fatos pertinentes, resultados negativos e declarações sobre o assunto devem ser incluídos, assegurando a completeza dessas informações.

Os critérios de apresentação do próprio sítio, dos links, dos links do design, da interatividade e dos anúncios apresentam indicadores detalhados no quadro 1, que também devem ser atenciosamente examinados.

Vale ressaltar que a qualidade da informação tem sido amplamente definida na literatura especializada, e, do contexto deste trabalho, destacam-se os critérios que estão em consonância com o modelo apresentado pelo Hiti, os quais têm sido validados por inúmeras instituições e grupos de especialistas.

Em um artigo de revisão, Molineux \& Williams (2000) analisam os estudos publicados na literatura que medem as características da Internet sob os seguintes aspectos: a avaliação da Internet, suas vantagens em relação à atualização das informações e as desvantagens relacionadas com a impermanência dos textos e endereços; o fluxo de usuários e quantitativos de dados circulantes, com ênfase em fontes de informação concentradas nos Estados Unidos.

Marchand (1990), apud Barbosa 1999), apresenta as cinco formas alternativas para definir qualidade da informação, sob os seguintes pontos de vista: de sua transcendência, de seu usuário, de seu produto, da sua produção e do seu valor. $\mathrm{Na}$ abordagem transcendente, presume-se que a qualidade da informação é absoluta e reconhecida universalmente. $\mathrm{Na}$ abordagem baseada no ponto de vista do usuário, está diretamente relacionada com as diferentes necessidades de informação de cada pessoa. A abordagem baseada no produto considera qualidade da informação em termos precisos e identificáveis relacionados com as características dos próprios produtos de informação. A abordagem baseada na produção define qualidade em termos de conformidade com as exigências, e a baseada em valores define a qualidade, focalizando o conceito de uso, redução de "ruído", adaptabilidade, economia de tempo e de custo, e a própria qualidade da informação.

Olaisen (1990), apud Nehmy \& Paim (1998), ressalta os fatores de qualidade da informação eletrônica, que são agrupados em quatro categorias: a primeira, denominada qualidade cognitiva, inclui os indicadores de credibilidade, relevância, confiança, validade e significado no tempo, e assemelhando-se à categoria apresentada pelo Hiti de credibilidade. A segunda categoria, denominada qualidade do desenho da informação, incorpora fatores referentes à forma, flexibilidade e seletividade, idem à categoria de links do Hiti. A terceira categoria de Olaisen se refere aos fatores relacionados ao produto de informação, que se assemelha à categoria de conteúdo do Hiti. A quarta categoria referese à qualidade da transmissão, que inclui o critério de acessibilidade incluído na categoria design do Hiti, todas citadas no quadro 1. A ênfase é dada ao conjunto destes fatores e de seu inter-relacionamento, configurando o processo de qualidade da informação.

No modelo de Kahn, Strong \& Wang (1997), a qualidade da informação pode ser definida nas suas quatro dimensões: relevância, interpretabilidade, credibilidade e reputação. Este modelo leva em conta a dimensão social da qualidade da informação sob o ponto de vista do usuário e inclui critérios semelhantes aos do Hiti. Em trabalho posterior, publicado em 2002, esses autores testam o modelo e aplicam a metodologia em um estudo de caso.

Lancaster (1989, cap. 4) definiu critérios específicos pelos quais os usuários avaliam os sistemas de recuperação, incluindo, entre outros, os que se referem à qualidade da informação, e que abordam cobertura, recuperação, precisão, novidade e confiabilidade do dado, proporcionando de certa maneira uma uniformidade com alguns dos critérios anteriores.

A questão da credibilidade dos sítios na Web está se tornando uma importante e crescente área de estudos, envolvendo não somente os profissionais de informação, mas também os de informática e profissionais advindos de inúmeras outros campos de atividades. Na tradicional Universidade de Stanford, na Califórnia, um grupo de pesquisadores do Persuasive Technology Laboratory*

\footnotetext{
* Stanford University. Stanford Persuasive Technology Laboratory. Disponível em: < http://www.webcredibility.org/ Acesso em 26 dezembro 2002.
} 
vem desenvolvendo pesquisas, desde 1998, sobre o problema de credibilidade das informações disponibilizadas na Web.

O registro na literatura de documentos sobre a avaliação de fontes eletrônicas, segundo Mostafa \& Terra (1998), constitui-se predominantemente dos cinco critérios de avaliação de fontes impressas, a saber: acuidade, autoridade, objetividade, atualização e cobertura. Afirmam as autoras que "com esses critérios quer-se assegurar a confiabilidade da fonte em termos das credenciais de autor e editor, somando-se os elementos pré-textuais como autor, título, editora e data da edição". Observa-se, portanto, que grande parte dos critérios apresentados na literatura especializada obedece a um consenso sobre os questionamentos básicos relacionados com o processo de comunicação científica, também mencionados por Sielberg et alii (1997).

\section{ESTUDOS SOBRE A QUALIDADE INFORMAÇÃO EM SAÚDE NA WEB}

$\mathrm{Na}$ área de saúde, existe um consenso internacional sobre a questão da qualidade da informação por parte de instituições governamentais, não-governamentais, associações de classe, profissionais de saúde e profissionais das mais diversas áreas afins, como atestam os estudos e critérios de qualidade de inúmeras instituições, podendo ser citados, entre outros, o grupo de pesquisa da Stanford University*; do Centre Hospitalaire Universitaire de Rouen ${ }^{* *}$; do Health on the Net Foundation ${ }^{* * *}$ e o Projeto DISCERN ${ }^{* * *}$, da Universidade de Oxford.

A confiabilidade das informações oferecidas na Web tem sido objeto de editoriais de periódicos científicos em diversas áreas e especialmente, na de saúde, advertindo seus leitores para a necessidade de precaução no que diz respeito às informações especializadas. Apesar dos aspectos positivos inerentes à própria diversidade de informações disponibilizadas para um público exponencialmente crescente, os instrumentos para avaliação dessas informações encontram-se em fase de definição e precisam ser constantemente reavaliados e

* Stanford University. The Web Credibility Research. Disponivel em: < http://www.webcredibility.org/ > Acesso em 26 dezembro 2002.

** NetScoring: critères de qualité de l'information de santé sur l'Internet. Rouen, Centre Hospitalaires Universitaire de Rouen, 1999.

*** Health on the Net (HON) Foundation, instituição com sede em Genebra na Suiça e que desde 1995, certifica páginas na Web. Disponível em < http://www.hon.ch >

**** DISCERN, disponivel em < http://www.discern.org.uk > readaptados, diante das transformações tecnológicas e por exigência das constantes mudanças no próprio ambiente da Internet.

Em editorial publicado no periódico Journal of the Americam Medical Association (Jama) em abril de 1997, Sielberg et alii (1997) registraram os primeiros critérios para avaliar, controlar e garantir a qualidade da informação médica na Web. Citados exaustivamente na literatura sobre qualidade de informação em saúde, os autores alertaram para as dificuldades inerentes à disseminação da informação incompleta, enganosa, sem acurácia sobre todas as áreas do conhecimento, e não apenas na área médica. Ressaltaram a importância dos critérios tradicionais de publicação na ciência e as regras que têm sido mantidas por mais de cinco séculos, desde a invenção da imprensa, salientando que os padrões pelos quais a ciência divulga novos conhecimentos diferencia, por exemplo, um editorial de uma propaganda, a educação de uma promoção e a evidência científica de uma opinião pessoal. Os que seguem estes padrões desenvolvem uma identidade respeitada, estabelecem um nível de confiança com seus leitores e servem como fórum de excelência para o avanço do conhecimento científico beneficiando o público em geral. Partindo deste princípio, sugerem que o mesmo núcleo de critérios de qualidade básicos que auxiliam os usuários a navegar na informação impressa tradicional pode ser aplicado no mundo digital.

Pandolfini e Bonati (2002) e Impicciatore et alii (1997) relatam um dos primeiros estudos publicados sobre avaliação da qualidade da informação em saúde na Web. Objetivando a avaliação da credibilidade da informação em saúde na Web e para auxiliar os usuários leigos a encontrar as informações em saúde com qualidade, os autores utilizaram uma metodologia qualitativa e quantitativa para análise do tema: tratamento domiciliar da febre em crianças. Após a execução da busca no Yahoo e no Excite, cruzaram os resultados obtidos, comparandoos com dados de publicações especializadas sobre febre na prática pediátrica. Os resultados encontrados apresentaram inconsistências médicas, e os autores, reconhecendo a complexidade do processo de avaliação, concluem que existe uma necessidade premente em se verificar a informação na Web sobre cuidados com a saúde dirigida ao público leigo, principalmente no que se refere à consistência, acurácia e completeza. Os critérios de avaliação aplicados evidenciam que o monitoramento da informação em saúde na Web deve ser implementado devido à ausência dos critérios de acurácia, completeza e consistência no conteúdo das páginas e informações disponibilizadas. 
Em estudo sobre a informação na área de saúde, Wyatt (1997) utilizou metodologia qualitativa e quantitativa para avaliar a qualidade desta informação. Tomando os critérios de qualidade propostos por Sielberg et al. (1997) e referendados parcialmente por Impicciatore et al. (1997), o estudo provocou uma reflexão em especialistas da área sobre as facilidades oferecidas na disseminação da informação na Web e a responsabilidade dos provedores desta informação. Os critérios investigados incluíram a acurácia das páginas da Web, comparandoas com evidências médicas. A atualização das páginas, o design e a correta forma de escrita foram também analisados, sobressaindo-se as categorias dos critérios de credibilidade, conflitos de interesse, autoria e credenciais do autor e do financiador; conteúdo e estrutura da página Web, além da citação das referências consultadas e da cobertura de assunto, todas estas constantes do conjunto de categorias especificadas pelo Healt Information Technology Institute (Hiti).

Jadad e Gagliardi (1998) empreenderam uma revisão sobre os critérios para avaliar a qualidade da informação em saúde veiculada na Web, com o objetivo de identificar os critérios utilizados e de estabelecer o grau de validade dos mesmos. Neste estudo foram identificados 47 critérios, também denominados instrumentos de avaliação pelos autores, sendo que 14 descreviam como foram desenvolvidos, cinco ofereciam instruções para uso e nenhum relatou as medidas de construção e validação do próprio instrumento de avaliação. A revisão demonstrou que muitos destes critérios foram desenvolvidos de forma incompleta, porém estavam sendo utilizados para avaliar a informação em saúde na Web. Em estudo posterior, publicado em 2002, os autores retornaram ao problema, revisando os critérios para avaliação das páginas Web em saúde, identificando, após cinco anos da primeira revisão, 51 novos critérios não mencionados anteriormente, nos quais os autores destacam as iniciativas institucionais para avaliação da informação em saúde.

Em um levantamento para avaliação da qualidade da informação em saúde na Web, Weiler \& Pealer (1998) apresentaram critérios e indicadores para auxiliar na identificação de páginas Web com credibilidade. Utilizando a metodologia de pesquisa documental, dividiram os resultados da busca exaustiva na Internet e em fontes tradicionais em duas categorias: uma relacionada com a página e outra com o conteúdo da informação. A categoria relacionada com a página da Web incluía apresentação do texto, acessibilidade, navegabilidade, disponibilidade de mecanismo de busca interno, links, interatividade, atualização do site legend, que deveria conter o título, endereço, autores e suas afiliações e endereço para contato, o responsável pela página, tipo de página, se comercial, governamental e outras, última data de atualização e dados de direito autoral, entre outros dados.

Em uma pesquisa utilizando método quantitativo, Oerman e Pasma (2001) basearam-se nos critérios do Hiti para avaliar cerca de dez páginas da Web para os consumidores de saúde sobre um tema específico: informação sobre assistência à saúde. Os autores partiram da premissa de que as páginas da Web, especializadas ou não, por vezes não oferecem os padrões de qualidade fundamentais para a disseminação da informação, apresentando dados incorretos e de procedência duvidosa. Por este motivo, os critérios de qualidade estabelecidos pelo Hiti foram testados para o tema selecionado em três dos maiores portais da Web especializados em saúde. $O$ estudo piloto foi desenvolvido junto a 40 consumidores com acesso pessoal à Web, de suas residências ou de bibliotecas públicas. Estes consumidores avaliaram cada documento, respondendo sim ou não às questões sobre as páginas previamente definidas, segundo os critérios do Hiti no documento Information Quality Tool.

Com o objetivo de estabelecer um núcleo de critérios de qualidade da informação em saúde na Web, a Comissão das Comunidades Européias (2002) utilizou o método quantitativo em pesquisa para definição de um conjunto de critérios de qualidade, para uso dos Estados membros e para o público em geral.

Durante evento realizado em 2001 - eEurope - an Information Society for All - 60 congressistas foram convidados a participar de uma consulta via Web, disponibilizada durante os meses de agosto a novembro de 2001. Os congressistas incluíam representantes da área industrial, médica, dos Estados-membros da Comunidade Européia e conferencistas da área de ética da informação em saúde. A consulta via Web identificou um conjunto de critérios de qualidade que incluíam, dentre outros: transparência e honestidade, autoridade, privacidade e proteção dos dados, atualização da informação, política editorial e acessibilidade. $O$ conjunto de critérios de qualidade estabelecidos baseou-se em consenso entre os diversos especialistas participantes, de autoridades na área de saúde e de usuários potenciais. $\mathrm{O}$ estudo gerou uma expectativa de que as autoridades em saúde nacionais e regionais, as associações de profissionais de saúde e as instituições que disponibilizam suas páginas na Web venham a implementar os critérios de qualidade propostos. 


\section{Ilza Leite Lopes}

Estudos que identificam como os usuários recuperam e determinam a qualidade da informação em saúde na Web são escassos, porém necessários. Neste sentido, a contribuição de Eysenbach \& Kohler (2002) veio preencher esta lacuna, em estudo qualitativo usando técnicas de grupo focal, de observação e de entrevistas aplicadas pós-buscas. Recrutados em anúncios de jornais, 21 usuários da Web participaram de três sessões de grupo focal, como voluntários. Cada sessão foi coordenada por um moderador e demorou de duas a três horas. O objetivo da utilização do método de grupo focal foi o de identificar as necessidades, expectativas e problemas dos usuários da informação em saúde na Web, com ênfase na avaliação destes em relação à qualidade da informação recuperada. O método de observação das buscas com temas predeterminados tinha por objetivo identificar como os usuários pesquisam na Web, se são bem-sucedidos, e como eles assinalam os resultados considerados de qualidade sobre a informação recuperada. As entrevistas foram aplicadas em 17 participantes do método de estudo observacional, imediatamente após as buscas realizadas na Web, para extrair deles os processos de decisão e os critérios de seleção de uma página particular na Web.

Objetivando estabelecer uma estrutura metodológica sobre os estudos que avaliam a qualidade da informação em saúde na Web, Eysenbach et alii (2002) analisam e comparam o rigor do método científico utilizado nestes estudos. A bibliografia citada neste artigo de revisão registra 122 referências e inclui documentos publicados em periódicos tradicionais, eletrônicos e em páginas da Web. Foram extraídas as características principais destes estudos, o domínio da área de saúde coberto, as estratégias de busca utilizadas e os métodos e critérios utilizados para determinação da qualidade da informação em saúde na Web. Os critérios de qualidade mais freqüentemente usados foram os de acurácia, completeza, legibilidade, design, informações do provedor da página e as referências bibliográficas.

Na área de jornalismo e comunicação da Ohio State University, pesquisadores desenvolvem projetos de pesquisa voltados para o problema do uso expressivo da Internet como meio de busca de informação, principalmente na área da saúde. Eastin (2001) salienta que os profissionais médicos não são necessariamente os autores das informações disponíveis na Web. Este fator cria a possibilidade de tal informação não oferecer garantia de credibilidade conforme os critérios tradicionais de comunicação científica e a validação pela revisão dos pares. A metodologia utilizada nesse estudo aplicou dois temas de busca na área médica (sífilis e
HIV) em sítios gerais e especializados, e os resultados indicaram que o nível de credibilidade maior adveio da consulta aos sítios especializados, principalmente pelo fato de estas informações serem de autoria explicitada nesses sítios.

Organismos nacionais* e internacionais como o Hiti** alertam para a oferta de informação em saúde sem o aval que a comunicação científica proporciona, devido aos riscos naturais proporcionados por informantes nãoqualificados para prover informações em saúde para a população em geral. Por esse motivo, alguns destes organismos internacionais já desenvolveram instrumentos de certificação da qualidade da informação disponível na Web e qualificam essas páginas colocando um selo de validade para aquelas que atendem aos critérios de qualidade definidos para informação na área de saúde.

Neste contexto, os paradigmas anteriormente existentes de legitimação da informação pela revisão dos pares parecem estar sendo substituídos por novos paradigmas, alguns deles voltados para a certificação da informação, sendo que várias instituições definiram selos específicos para validação das páginas Web na área de saúde, tais como a HON Foundation na Suíça, com seu selo e Código de Conduta (HONcode) ${ }^{* * *}$ para os sítios da Web em medicina e saúde, e o Centre for Health Information Quality (CHIQ) ${ }^{* * *}$, com um selo de validade para o Reino Unido.

\section{PROPOSTAS BRASILEIRAS SOBRE A QUALIDADE DA INFORMAÇÃO EM SAÚDE}

No que concerne à área de saúde, como é consenso geral, a maioria da população brasileira tem na automedicação um processo natural e atávico de sobrevivência e, por esse motivo, a facilidade de uso da Internet na própria residência promove uma natural busca de informações sobre saúde na Web. Este, porém, parece ser um comportamento universal; na Alemanha, Eysenbach (2000), em análise sobre o consumidor de informação em saúde, salienta que, assim como os profissionais de

* CFM - Conselho Federal de Medicina, Resolução n 1643 de 2002. Disponível em: < http://www.cfm.org.br/ResolNormat/ Numerico/1643_2002.htm > Acesso em 29 outubro 2002.

** Hiti - Disponível em: < http://www.hitiweb.mitretek.org > Acesso em 13 março 2002.

*** HON Code - Disponível em < http://www.hon.ch/HONcode/ Portuguese $>$ Acesso em 17 abril 2003

**** CHIQ - Centre for Health Information Quality . Disponível em: $<$ http://www.hfht.org/chiq/quality.htm Acesso em 20 novembro 2002 . 
saúde necessitam conviver com as modernas tecnologias de informação, os pacientes destes profissionais são efetivos consumidores desta informação, utilizando o mesmo recurso para buscar as mais variadas informações sobre questões pessoais e familiares de saúde, percepção dos sintomas de uma doença e o comportamento da própria doença, além de conhecimentos genéricos sobre os tratamentos recomendados pelos especialistas.

No Brasil, os órgãos que regulamentam a ética médica, como o Conselho Federal de Medicina (CFM), os conselhos regionais do Rio de Janeiro e de São Paulo e a Associação Médica Brasileira, além de numerosas outras instituições envolvidas com os aspectos educacionais e profissionais na área de saúde, estão procurando agir no sentido de tentar a regulamentação das informações divulgadas na Web.

O manual de princípios éticos para sitios de medicina e saúde na Internet*, do Conselho Regional de Medicina de São Paulo (Cremesp) definiu princípios éticos e critérios de conduta das páginas na Internet, apresentando um conjunto de conceitos para aplicações na área de medicina e saúde semelhantes em alguns pontos ao Hiti e a HON Foundation, incluindo: transparência, honestidade, qualidade, consentimento livre e esclarecido, privacidade, ética médica e responsabilidade e procedência. Em relação à qualidade, afirmam que:

"A informação de saúde apresentada na Internet deve ser exata, atualizada, de fácil entendimento, em linguagem objetiva e cientificamente fundamentada. Da mesma forma, produtos e serviços devem ser apresentados e descritos com exatidão e clareza. Dicas e aconselhamentos em saúde devem ser prestados por profissionais qualificados, com base em estudos, pesquisas, protocolos, consensos e prática clínica. Os sites com objetivo educativo ou científico devem garantir autonomia e independência de sua política editorial e de suas práticas, sem vínculo ou interferência de eventuais patrocinadores. Deve estar visível a data da publicação ou da revisão da informação, para que o usuário tenha certeza da atualidade do site. Os sites devem citar todas as fontes utilizadas para as informações, o critério de seleção de conteúdo e a política editorial do site, com destaque para nome e contato com os responsáveis."

\footnotetext{
* Cremesp. Manual de princípios éticos para sítios de medicina e saúde na Internet. Disponivel em < http://www.saudeinformacoes.com.br/ institucional_cremesp.asp > acesso em 16/04/2003
}

A preocupação do Cremesp fica bem evidenciada pelos pontos principais mencionados sobre a questão da qualidade da informação, que são semelhantes aos estabelecidos como critérios de avaliação de qualidade propostos pelo Hiti e pela HON Foundation.

Além disso, o Centro de Vigilância Sanitária (CVS) do governo do estado de São Paulo, no contexto do comércio eletrônico de medicamentos e de produtos e serviços de saúde, traduziu e adaptou para o Brasil um documento da Organização Mundial de Saúde, intitulado Guia para encontrar informações seguras*, cujo objetivo é fornecer ao usuário condições de avaliação dos conteúdos de páginas na Web. Este guia sugere a averiguação das informações dessas páginas, dentre outras, com aquelas relacionadas com a autoridade do autor e da instituição, dos patrocinadores, os objetivos e a data de publicação da informação, consolidando, portanto, os critérios de avaliação sugeridos na literatura especializada.

Observa-se ainda que, no Brasil, o Conselho Federal de Medicina, os conselhos regionais de medicina do Rio de Janeiro e de São Paulo e outras instituições estão se mobilizando para regular a apresentação das páginas Web em medicina e saúde no país, definindo conceitos e ações para preservar a credibilidade da informação em saúde.

\section{QUALIDADE DA INFORMAÇÃO EM SAÚDE: INICIATIVAS DE ORGANISMOS INTERNACIONAIS}

Além dos autores acadêmicos que apresentam sugestões de critérios para avaliação da informação, organismos nacionais e internacionais vêm desenvolvendo instrumentos reguladores e códigos de conduta, visando ao estabelecimento de padrões éticos para a informação em saúde disponibilizada na Internet. Constituídas por profissionais de saúde e grupos multidisciplinares, estas instituições, desde 1996, elaboram e definem ações que se concretizam em diretrizes respeitadas e seguidas pela comunidade em saúde.

Uma das primeiras iniciativas para avaliação da qualidade da informação em saúde foi realizada em 1996 pela British HealthCare Internet Association (BHIA)**, organização sem fins lucrativos, voltada para as tecnologias aplicadas à Internet. Os membros da BHIA são médicos, editores, Web designers, provedores de

\footnotetext{
* Disponível em http://www.cvs.saude.sp.gov.br/medical.htm > Acesso em 19 maio 2003.

** BHIA. Disponível em: < http://www.bhia.uk > Acesso em 13 março 2002
} 


\section{Ilza Leite Lopes}

informação, profissionais de tecnologia da informação, professores, pesquisadores e profissionais de agências governamentais, somando ao todo 120 membros, que têm por objetivo o aprimoramento da qualidade da informação médica na Web.

Com ênfase na certificação de páginas Web em saúde, surgiu em 1996, em Genebra, na Suíça, uma organização sem fins lucrativos denominada Health On the Net $(\mathrm{HON})$ Foundation *. Esta fundação, que tem por objetivo a implementação de projetos na Internet e em telemedicina que beneficiem a área de saúde, recebe o apoio do Ministério de Saúde do Estado, além de doações de outras fontes, incluindo o Swiss Institute for Bioinformatics e a empresa Sun MicroSystems.

A Fundação HON confere o selo de certificação de qualidade para páginas Web, desde que as páginas atendam aos oito princípios básicos, com padrões éticos estabelecidos pela fundação em conjunto com médicos, provedores de informação, cidadãos, pacientes e Webmasters. Estes princípios éticos estão definidos no Código de Conduta (HONCode) para páginas Web em medicina e saúde e se referem à autoridade, complementaridade, confidencialidade, atribuições, justificativas, transparência na propriedade, transparência do patrocínio e honestidade da publicação e da política editorial. $\mathrm{O}$ código de ética da Fundação HON tem sido apoiado por inúmeras instituições, incluindo cerca de 44 empresas de seguro médico, oriundas de 23 países, que avalizam os princípios que asseguram a qualidade da informação.

O programa desenvolvido no Reino Unido em 1996, pelo Joint Information Services Commitee (JISC) denominado OMNI - Organizing Medical Networked Information, tem como objetivo oferecer acesso às informações da Web, pelo Portal BIOME**. Estas informações são classificadas pelos critérios de avaliação de qualidade desenvolvidos pelo OMNI - Advisory Group on Evaluation Criteria e abrangem informações sobre medicina alternativa. Os membros do OMNI são constituídos por médicos e especialistas em diversas áreas de saúde de inúmeras instituições no Reino Unido.

\footnotetext{
* HON Foundation. Disponível em: < http://www.hon.ch > Acesso em 17 abril 2002.

** BIOME disponível em http://www.biome.uk acesso em 22/4/ 2002.
}

Em um projeto desenvolvido com o apoio da Agency for HealthCare Policy and Research (AHCPR) em 1997, o Health Information Technology Institute (Hiti) elaborou um documento definindo critérios para avaliação da qualidade da informação em saúde na Internet*.

Com a participação de profissionais da área de saúde oriundos de órgãos governamentais e privados, foram estabelecidas sete categorias de critérios de avaliação com seus respectivos indicadores, apresentados no quadro 1 (pág. 82), e que têm norteado estudos semelhantes em vários países.

$\mathrm{Na}$ França, o Ministério de Saúde e Solidariedade** traduziu e divulga todos os critérios de avaliação do Hiti, validando de certa forma o empenho desta instituição no estabelecimento de diretrizes para aprimorar a qualidade da informação em saúde. Com base na análise criteriosa de páginas Web em saúde e no consenso de uma equipe interdisciplinar na área, foi recomendada uma série de critérios, tais como credibilidade da fonte, contexto, atualidade, utilidade, processo de revisão editorial, acurácia do conteúdo, evidências em que se baseia, citação das fontes originais, objetivo do documento e público-alvo. Apresentam-se, ainda, critérios de qualidade quanto ao design, links, organização lógica, arquitetura e outros. Estes critérios, estabelecidos no auge da criação de páginas Web, serviram de marco consensual para diversas instituições na elaboração de critérios similares que avaliassem a qualidade da informação em saúde na Web.

Outra iniciativa internacional de controle da qualidade da informação em saúde é o Projeto Discern***, desenvolvido em 1999 pelo UK National Health Service Executive Research and Development Programm, e que está baseado na Universidade de Oxford, no Instituto de Ciências da Saúde. Este projeto tem como objetivo principal possibilitar o julgamento sobre a qualidade da informação em tratamentos médicos utilizando um conjunto de critérios apresentados em forma de questionário on-line. Constituído por profissionais de saúde e pesquisadores da Universidade, o Discern oferece uma oportunidade de avaliar a qualidade da informação em saúde no Reino Unido.

\footnotetext{
* Hiti - Criteria for Assessing Quality Information on the Web. Disponível em : http://hitiweb.mitreteki.org/docs/criteria.html > Acesso em 13 março 2002.

** Ministére de Sante et Solidarieté. Disponível em < http:// www.sante.gouv.fr > Acesso em 9 março 2003.

*** DISCERN on the Net. Projeto da Universidade de Oxford, disponível em < http://discern.org.uk > Acesso em 9 março 2003.
} 
As iniciativas internacionais não se esgotam no presente estudo, mas vale destacar, dentre outras, as seguintes instituições: US Department of Health and Human Services*, American Accreditation HealthCare Commission**, Internet HealthCare Coalition***, Centre for Health Information Quality - CHIQ ${ }^{* * *}$ e National Health Service ${ }^{* * * *}$, que apresentam contribuições para a possível regulamentação e controle de qualidade das informações disponibilizadas nas páginas da Web.

\section{CONSIDERAÇÕES FINAIS}

A literatura especializada constata que o crescimento da Internet nos últimos anos refletiu-se não apenas no aumento do número de servidores, documentos, serviços e usuários, mas também na sua diversificação em oferta de serviços, como transferência de arquivos, correio eletrônico, além da própria Web. Assim, os novos tipos de documentos e serviços (documentos multimídia, serviços interativos e outros) e a própria utilização da Internet para além da comunidade acadêmica provocaram algumas conseqüências fundamentais diretamente relacionadas com esse crescimento: a dificuldade de acompanhamento das mais recentes novidades e desenvolvimentos e a complexidade em se avaliar a qualidade da informação recuperada, diante da descentralização do processo de produção da informação e da inexistência de mecanismos de controle de qualidade dessa informação.

Esta revolução nos meios de disseminação da informação científica tem provocado posicionamentos de organismos governamentais e não-governamentais, de associações de classe, de médicos e outros profissionais de saúde, visando a uma possível regulamentação nas informações veiculadas na Web, com iniciativas diversificadas de controle de qualidade sobre as mesmas. Segundo Kuhn (1970), o período de revolução científica é, neste sentido,

* US Dept. of Health \& Human Services, com o Diretório Healthfinder, disponível em: < http://healthfinder.com > Acesso em 17 abril 2003

** American Accreditation HealthCare Commission apresenta um programa com critérios para desenvolvimento de conteúdo de Wesites. Disponível em: < http://www.urac.org > Acesso em 17 abril 2003.

*** Internet HealthCare Coalition, desenvolveu um Código de Ética para avaliação dos Websites. Disponível em : < http:// www.ihealthcarecoalition.org > Acesso 9 março 2003.

**** Centre for Health Information Quality - CHIQ, desenvolveu um Selo de qualidade para Websites. Disponível em: < http:// www.chiq.uk > Acesso em 7 março 2003.

***** National Health Service - NHS. Desenvolveu um diretório de fontes de informação em Saúde. Disponível em: < http:// nhsdirect.nhs.uk > Acesso em 7 março 2003. um período de mudança de paradigmas, e os diferentes paradigmas irão considerar diferentes tipos de questionamentos como legítimos ou significativos. Assim, a avaliação da produção científica antes consagrada pela revisão dos pares e por instrumentos aceitos por toda a comunidade científica, passa por uma fase de transição sucessiva, em que novas vertentes de inovações para validação da informação científica vão sendo desenvolvidas, com o objetivo de contornar a questão da qualidade da informação.

Paralelamente, a literatura especializada na questão específica da avaliação do conteúdo das páginas na Web tem variado de recomendações pontuais, como as apresentadas por Alexander \& Tate (1996), Harris (1997), Weiler et alii (1998) e Wyatt (1997), indo aos mais teóricos como Khan et alii (1997), Tillman (1997) e Fogg et alii (2001, 2002), e passando por questões práticas recomendadas, dentre outros, por Sielberg et alii (1997), Jadad \& Gagliardi (1998), o Hiti (1999), e Europe (2001) e Eysenback et alii. ( 2002).

As questões em torno da natureza, da estrutura e do valor da avaliação realizada pelos pares para publicação em periódicos científicos são, de certa forma, uma garantia de certificação da qualidade acadêmica, o que não vem ocorrendo plenamente no modelo eletrônico. Neste sentido, a publicação eletrônica, por se constituir em método rápido de disseminação dos resultados para a comunidade científica, proporciona um questionamento sobre a mudança no papel de revisão pelos pares, diante dos novos modelos paradigmáticos de verificação da qualidade da informação, que incluem os códigos de ética e/ou de conduta e os selos de certificação de qualidade nas páginas da Web.

Artigo recebido em 17-10-2003 e aceito para publicação em 24-04-2004. 


\section{Ilza Leite Lopes}

\section{REFERENNCIAS}

ALEXANDER, J.; TATE, M. A. Evaluating web resources: checklist for a informational web page. Disponível em: 〈http://www2.widener.edu/ Wolfgram-Memorial-Library/ webevaluation/inform.htm>. Acesso em: 12 mar. 2002

BARBOSA, Elza M. F. Erros e acurácia em bases de dados bibliográficas nas áreas de Ciência e tecnologia. 1999. 150 f. Tese. (Doutorado em Ciência da Informação) - Departamento de Ciância da Informação da Universidade de Brasília, Brasília, 1999.

CENTRO DE VIGILÂNCIA SANITÁRIA (São Paulo, SP). Venda de medicamentos, produtos e serviços de saúde via Internet: guia para encontrar informaçoes seguras. Disponível em: <http:// www.cvs.saude.sp.gov.br/medical.htm>. Acesso em: 19 maio 2003.

EASTIN, M. S. Credibility assessments of online health information: the effects of source expertise and knowledge of content. JCMC, v. 6 , n. 4, p. 10-26, July, 2001. Disponível em: <http://www.ascusc.org/ jcmc/vol6/issue4/eastin.html>. Acesso em: 26 dez. 2002.

EUROPE 2002: quality criteria for health related websites. JMIR, v. 4, n. 3, p. 15, 2002. Disponível em: <http://www.jmir.org/2002/3/ e15/index.htm>. Acesso em: 22 jan. 2003.

EYSENBACH, G.; KOHLER, C. How to consumers search for and appraise health information on the world wide web? Qualitative study using focus groups, usability tests, and in-depth interviews. BMJ, v. 324, p. 573-577, 2002.

. et al. Empirical studies assessing the quality of health information for consumers on the world wide web: a systematic review. JAMA, v. 287, n. 20, p. 2691-724, May 2002.

FOGG, B. J. Stanford guidelines for web credibility: a research summary from the Stanford Persuasive Technology Laboratory. [S. 1.] : Stanford University. 2002. Disponível em: <http:// www.webcredibility.org/guidelines.html>. Acesso em: 26 dez. 2002. . et al. What makes web sites credible? A report on a large quantitative study. CHI, v. 3, n. 4, Mar. 2001.

GAGLIARDI, A.; JADAD, A. R. Examination of instruments used to rate quality of health information on the internet: chronicle of a voyage with and unclear destination. BMJ, v. 324, p. 569-573, 2002.

HARRIS, R. Evaluating Internet research sources Disponível em: <http://www.virtualsalt,com/eval8it.htm>. Acesso em : 22 out. 2002. HEALTH INFORMATION TECHNOLOGY INSTITUTE. Agency for Health Care Policy and Research Criteria for Assessing the Quality of Health Information on the Internet. McLean, VA, 1999. Disponivel em: 〈http://www.hitiweb.mitretek.org/docs/criteria.html>. Acesso em: 13 mar. 2002.

IMPICCIATORE, P. et al. Reliability of health information for the public on the World Wide Web: systematic survey of advice on managing fever in children at home. BMJ, v. 314, p. 1875-1882, June 1997. Disponível em: <http://bmj.com.cqi/content/full/314/7098>. Acesso em: 14 jan. 2003.

JADAD, AR.; GAGLIARDI, A. Rating health information on the Internet: navigating to knowledge or to Babel? JAMA, v. 279, n., 8, p. 611-614, Feb. 1998
JONES, S. L.; COOK, C. B. Electronic journals: are they a paradigm shift? Online Journal of Issues in Nursing, v. 5, n. 1, Jan. 2000. Disponivel em: 〈http://www.nursingworld.org/topic11/ptc11_1.htm>. Acesso em: 13 jul. 2003.

KAHN, B. R.; STRONG, D. M; WANG, R. Y. A model for delivering quality information as product and service. In: CONFERENCE ON INFORMATION QUALITY, 1997. Proceedings... Cambridge, MA, [ : s. n.], 1997. p. 80-94.

Information quality benchmarks: product and service performance. Communications of the ACM, v. 45, n. 4, p. 184-192, Apr. 2002.

KUHN, T. A estrutura das revoluções científicas. São Paulo : Perspectiva, 1970 .

LANCASTER, F.W. The measurement and evaluation of library systems. Arlington : Information Resource, 1989. Cap. 4, p. 140-164.

MARCHAND, D. Managing information quality. In: WORMELL, I. (Ed.). Information quality: definitions and dimensions. [S. 1.] : Taylor Graham, 1990. p. 7-17.

MOLYNEUX, R.; WILLIAMS, R. V. Measuring the Internet. ARIST, v. 34, p. 287-340, 1999-2000.

MOSTAFA, S. P.; TERRA, M. As fontes eletrônicas de informação: novas formas de comunicação e de produção do conhecimento. São Paulo em Perspectiva, v. 12, n. 4, p. 1-12, out./dez. 1998.

NEHMY, R. M. Q; PAIM, I. A desconstrução do conceito de qualidade da informação. Ciência da Informação, Brasília, v. 27, n. 1, p. 36-45, 1998. Disponível online em: < http://www.ibict.br>.

OERMAN, M. H ; PASMA, J. Evaluation by consumers of quality care information on the Internet. Journal Nursing Care Quality, v 15, n. 3, p. 50-58, 2001.

OLAISEN, J. Information quality factor and the cognitive authority of electronic information. In: WORMELL, I. (Ed.). Information quality: definitions and dimensions. [S. 1. : s. n., 2001?]. p. 91-121.

PANDOLFINI, C.; BONATI, M. Follow up of quality of public oriented health information on the world wide web: systematic reevaluation. BMJ, v. 324, p. 582-583, Mar. 2002. Disponível em: <http://bmj.com.cgi/content/full/324/7337 >. Acesso em: 16 jan. 2003.

SIELBERG, W. M.; LUNDBERG G. D.; MUSACCHIO R. A. Assessing, controlling and assuring the quality of medical information on the Internet... JAMA, v. 277, n. 15, p. 1244-1245, 1997. Disponivel em: http://gateway2.ovid.com/ovidweb.cgi >. Acesso em: 14 fev. 2003.

TILLMAN, H. Evaluating quality on the net. Disponivel em: http:// www.tiac.net/users/hoe/findqual.html. Acesso em: 22 out. 2002.

WEILER, R. M.; PEALER, L. N. A survey of criteria and indicators for evaluating the quality of health information on the world wide web. Disponível em:<http://www.hhp.ufl.edu/hse/faculty/rweiler/asha98/>. Acesso em: 24 fev. 2003.

WYATT, J. C. Measuring quality and impact of the World Wide Web. BMJ v. 314 15, June, 1997. Disponível em: <http://bmj.com/ cgoi/content/full/314/7098/1879>. Acesso em: 17 nov. 2002. 